\title{
Predicting Total Energy Consumption with Least Square Method of Legendre Polynomial

\author{
Lai-Bin $\mathrm{GAO}^{1, \mathrm{a}^{*}}$, Bo WANG ${ }^{1, \mathrm{~b}}$
}

${ }^{1}$ College of Information and Technology, Jilin Agricultural University, Changchun, 130118, China

$$
\text { awanggao911@163.com, bzhanghq_@126.com }
$$

${ }^{*}$ Corresponding author

\section{Keywords: The Least Square Method, Normal Equation, Legendre Polynomial.}

\begin{abstract}
Based on statistical data of National Statistical Bureau of China, and given the least-squares fitting of Legendre polynomial, the data of total energy consumption from 1978 to 2014 is analyzed by least squares method and Legendre polynomial least squares method respectively. The results showed that Legendre polynomial least squares fitting method is excellent and the data of total energy consumption from 2015 to 2016 is predicted by this method.
\end{abstract}

\section{Introduction}

In the scientific research and production practice, the data is obtained from observation, prediction and calculation should be analyzed and processed, and then the conclusions concerning the problems can be found out. The polynomial least square method is an effective method. However, the accuracy and reliability of the least square method relies on the condition number of the coefficient matrix of its normal equation. In this article, Legendre polynomial is adopted as basis function, so as to obtain the small condition number of coefficient matrix of the normal equation of the least square method. With China's increasingly affluent urban, rural living modern and living energy consumption increasing every year, energy conservation has become important part of China's energy conservation. Therefore, the total energy consumption is predicted accurately is very important for socio-economic development.

\section{The Fundamental Principles of the Least Square Method}

\section{The Linear Least Square Fitting of General Polynomial[1]}

Let $\mathrm{Q}_{n}(x)=\operatorname{span}\left(1, x, \mathrm{~L}, x^{n}\right)$ denote the linear space formed by, then for any $p_{n}(x) \in \mathrm{Q}_{n}(x)$, there are $a_{0}, a_{1}, \mathrm{~L}, a_{n}$, which satisfy the following equation

$$
p_{n}(x)=a_{0}+a_{1} x+\mathrm{L}+a_{n} x^{n}
$$

Supposing there is a set of data $\left\{\left(x_{i}, y_{i}\right)\right\}_{i=1}^{m}$, where $x_{1}, x, \mathrm{~L}, x_{m}$ are different each other. Then the coefficients $a_{0}, a_{1}, \cdots, a_{n}$ will be found, which satisfy as follows

$$
E_{2}\left(a_{0}, a_{1}, \mathrm{~L}, a_{n}\right)=\sum_{i=1}^{m}\left|y_{i}-p_{n}\left(x_{i}\right)\right|^{2}=\sum_{i=1}^{m}\left[\sum_{j=0}^{n} a_{j} x_{i}^{j}-y_{i}\right]^{2}
$$

The method is called the least square, the functions $1, x, \cdots, x^{n}$ are the basis of the linear least square, $E_{2}\left(a_{0}, a_{1}, \cdots a_{n}\right)$ is called the residual sum of squares. It is well known that the least square problems must have a solution and it is the solution the linear equation. 


$$
\begin{aligned}
P A & =\left[\begin{array}{cccc}
s_{0} & s_{1} & \mathrm{~L} & s_{n} \\
s_{1} & s_{2} & \mathrm{~L} & s_{n+1} \\
\mathrm{M} & \mathrm{M} & & \mathrm{M} \\
s_{n} & s_{n+1} & \mathrm{~L} & s_{2 n}
\end{array}\right]\left[\begin{array}{c}
a_{0} \\
a_{1} \\
\mathrm{M} \\
a_{n}
\end{array}\right]=\left[\begin{array}{c}
u_{0} \\
u_{1} \\
\mathrm{M} \\
u_{n}
\end{array}\right], \\
A & =P^{-1} U, \quad \text { where } \mathrm{s}_{\mathrm{k}}=\sum_{i=1}^{m} x_{i}^{k}, u_{k}=\sum_{i=1}^{m} y_{i} x_{i}^{k}, \quad k=0,1, \mathrm{~L}, n \text {. The linear equation is called the }
\end{aligned}
$$

normal equation of the least square. So the accuracy of the linear least square relies on the accuracy of normal equation. The solution of the linear equation is unreliable while the condition number of the coefficient matrix of the linear equation ${ }^{[3]}$ is very big. For example:

$$
\left[\begin{array}{lll}
1 & 2 & 3 \\
4 & 5 & 6 \\
7 & 8 & 9
\end{array}\right]\left[\begin{array}{l}
x_{1} \\
x_{2} \\
x_{3}
\end{array}\right]=\left[\begin{array}{l}
12 \\
30 \\
48
\end{array}\right]
$$

The exact solution of the equation is $(2,2,2)$, but the solution obtained by using the Matlab is $(0,6,0)$ and the condition number of the coefficient matrix of the linear equation is $1.55 \times 10^{18}$. It can be seen that the condition number of the coefficient matrix of the normal equation of general polynomial linear least square is big correspondingly with the consecutive natural numbers $\left\{x_{i}\right\}_{i=1}^{m}$.

\section{The Linear Least Square of Legendre Polynomial[2]}

The expression of the Legendre polynomial is that:

$$
\begin{aligned}
& P_{n}(t)=\frac{1}{2^{n} n !} \frac{d^{n}\left(t^{2}-1\right)^{n}}{d t^{n}}, \quad n=1,2, \cdots \\
& P_{0}(t)=1
\end{aligned}
$$

Moreover, the Legendre polynomial in the interval $[-1,1]$ can be obtained by the following recurrence relations

$$
\begin{aligned}
& P_{n+1}(t)=\frac{2 n+1}{n+1} t P_{n}(t)-\frac{n}{n+1} P_{n-1}(t) \quad n=1,2, \cdots \\
& P_{0}(t)=1 \quad P_{1}(t)=t
\end{aligned}
$$

By transformation, the Legendre polynomial can be obtained in the interval ${ }^{[a, b]}$ :

$$
\begin{aligned}
& t=\frac{2 x-(a+b)}{b-a}, \quad x \in[a, b], t \in[-1,1] \\
& \tilde{P}_{n}(x)=P_{n}(t)=P_{n}\left(\frac{2 x-(a+b)}{b-a}\right)
\end{aligned}
$$

Giving a set of data, $\left\{\left(x_{i}, y_{i}\right)\right\}_{i=1}^{m}$, and $a=x_{1}<x_{2}<\cdots<x_{m}=b$. Let $\tilde{P}_{k}(x), k=0,1, \cdots, n$ be the basis functions, there are $c_{0}, c_{1}, \cdots, c_{n} \in R$ such that

$$
\begin{aligned}
& \varphi(x)=c_{0}+c_{1} \tilde{P}_{1}(x)+\cdots+c_{n} \tilde{P}_{n}(x) \\
& E_{2}\left(c_{0}, c_{1}, \cdots c_{n}\right)=\sum_{i=1}^{m}\left|y_{i}-\varphi\left(x_{i}\right)\right|^{2}=\sum_{i=1}^{m}\left[\sum_{j=0}^{n} c_{j} \tilde{P}_{j}\left(x_{i}\right)-y_{i}\right]^{2}
\end{aligned}
$$

where $\tilde{P}_{j}=\left[\tilde{P}_{j}\left(x_{1}\right), \cdots \tilde{P}_{j}\left(x_{m}\right)\right]^{T}, j=0,1, \cdots, n, y=\left[y_{1}, \cdots y_{m}\right]^{T}, C=\left[c_{0}, \cdots, c_{n}\right]^{T}$. Therefore its 
normal equation of the least square is $G C=b$, where $G=\left[\left(\tilde{P}_{j}, \tilde{P}_{k}\right)\right],\left(\tilde{P}_{j}, \tilde{P}_{k}\right)$ refers to the inner product of $\tilde{P}_{j}$ and $\tilde{P}_{k}, b=\left[\left(y, \widetilde{P}_{0}\right), \cdots,\left(y, \tilde{P}_{n}\right)\right]^{T}$. The condition number of the coefficient matrix of this normal equation is very tiny, so the fitting accuracy and practicability is high.

\section{The Prediction of Total Energy Consumption}

\section{Fitting Function}

The data of total energy consumption from 1978 to 2014 is analyzed and the data of total energy consumption from 2015 to 2016 is predicted by the least square fitting method. Data from 1978 to 2014 is as Tab. (a).

Tab. (a) The data of total energy consumption from 1978 to $2012 \times 10^{7} \mathrm{~kg}$ (standard coal)

\begin{tabular}{cc|cc||cc}
\hline Year & Yield & Year & Yield & Year & Yield \\
\hline 1980 & 60275 & 1997 & 135909 & 2006 & 286467 \\
1985 & 76628 & 1998 & 136184 & 2007 & 311442 \\
1990 & 98703 & 1999 & 140569 & 2008 & 320611 \\
1991 & 103783 & 2000 & 145531 & 2009 & 336126 \\
1992 & 109170 & 2001 & 155547 & 2010 & 360648 \\
1993 & 115993 & 2002 & 169577 & 2011 & 387043 \\
1994 & 122737 & 2003 & 197083 & 2012 & 402138 \\
1995 & 131176 & 2004 & 230281 & 2013 & 416913 \\
1996 & 135192 & 2005 & 261369 & 2014 & 426000 \\
\hline
\end{tabular}

By using the Matlab mathematic software, the fitting result of the polynomial least square is as follows:

$$
p(x)=341.7294 x^{2}-1.3551 \times 10^{6} x+1.3435 \times 10^{9}
$$

The fitting result of Legendre polynomial least square is that Let $\mathrm{a}=1978, \mathrm{~b}=2014$ and $t=\frac{2 x-(a+b)}{b-a}$, then we have

$$
\varphi(t)=1.4991 \times 10^{5}+1.4234 \times 10^{5} t+0.6584 \times 10^{5} \times \frac{1}{2}\left(3 t^{2}-1\right)
$$

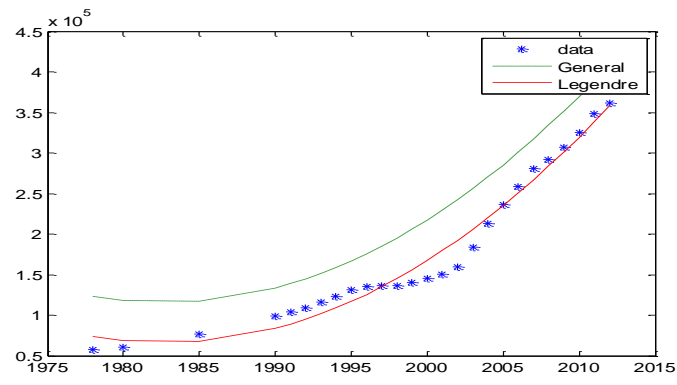

Fig.1 The actual fitting curve energy consumption from 1978 to 2012

It can be seen from Fig.1 that the effect of the general polynomial least square is bad, but the fitting result of the Legendre polynomial least square is more accurate. By calculating, we get the 
condition number of the coefficient matrix of the linear least square of general polynomial is $2.6472 \times 10^{22}$, but that of the Legendre polynomial is just 4.6746 .

\section{Evaluation on the Fitting Results}

\section{Evaluation on Unbiased Variance[5]}

The following formula is used to evaluate the unbiased variance

$$
M S E=\frac{1}{N} \sum_{i=1}^{N} e_{i}^{2}=\frac{1}{N} \sum_{i=1}^{N}\left(y_{i}-\hat{y}_{i}\right)^{2}
$$

where $\hat{y}_{i}$ is the fitting evaluation value of $x_{i}$. When using the unbiased variance to evaluate, the usually followed principle is the smaller the mean square error, the better the method used. The linear least square of general polynomial $M S E=2.7283 \times 10^{9}$, but the linear least square of the Legendre polynomial $M S E=2.1131 \times 10^{8}$.

\section{Determination of the Coefficient[4]}

The formula of the determination coefficient is as follows

$$
R^{2}=1-\frac{\sum\left(y_{i}-\hat{y}_{i}\right)^{2}}{\sum\left(y_{i}-\bar{y}\right)^{2}}
$$

In the formula, $\bar{y}$ is the average value of ${ }^{y_{i}}$.When using the determination coefficient $R^{2}$ to evaluate, usually we follow the principle that $R^{2}$ closes to 1 is the best method. the linear least square of general polynomial is $R^{2}=0.7368$, but the linear least square Legendre polynomial is $R^{2}=0.9729$.

\section{Conclusion}

Firstly, when analyzing the data of scientific research, the linear least square of the Legendre polynomial is better method.

Secondly, by establishing the Legendre polynomial least square model of the data of the total energy consumption from 1978 to 2012, energy consumption per capita from 2015 to 2016 can be predicted, which is $4.3114 \times 10^{12} \mathrm{~kg}, 4.4353 \times 10^{12} \mathrm{k} \mathrm{kg}$, respectively. Therefore, saving energy in our lives is a very important problem.

Thirdly, Residential energy conservation has become a part of important China's energy conservation by developing good saving habits, using of scientific methods of energy, China's 1.3 billion people can make an important contribution to energy conservation. For example: choosing energy-saving air-conditioning, one degree Celsius increase in summer air-conditioning, using energy-saving lamps, using energy-efficient refrigerator, etc.

\section{References}

[1] Yunshi Zhou, Liang Xun. Numerical Analysis [M].Beijing: Higher Education Press, 2008:131-135.

[2] Chengsun Lin. Numerical Analysis [M]. Beijing: Science Press .2007:174-178.

[3] ]HEATH MT. Scientific Computing [M].Beijing: Tsinghua University Press .2005:59-61.

[4] Shisong Mao, CHENG YM. Tutorial of Probability and mathematical Statistics [M]. Beijing: Higher Education Press .2004:418-419.

[5] Chenfang Ding. Application of Combination forecasting method in China's grain output prediction [J]. Research of Agricultural Modernization, 2007 (3): 101-103. 